\title{
A rare case of male pelvic squamous cell carcinoma of unknown primary origin presenting as perineal abscess and urethral stenosis
}

\author{
Massimiliano Creta ${ }^{1}$, Vincenzo Mirone ${ }^{2}$, Sergio Di Meo ${ }^{1}$, Roberto Buonopane ${ }^{1}$, Nicola Longo ${ }^{2}$, \\ Ferdinando Fusco $^{2}$, Nicola Rosario Forte ${ }^{3}$, Vittorio Imperatore ${ }^{1}$ \\ ${ }^{1}$ Unità Operativa di Urologia, Ospedale Buon Consiglio - Fatebenefratelli, Napoli, Italy; \\ ${ }^{2}$ Clinica Urologica, Università Federico II di Napoli, Napoli, Italy; \\ ${ }^{3}$ Unità Operativa di Anatomia Patologica, Ospedale Fatebenefratelli, Benevento, Italy.
}

\begin{abstract}
Summary Carcinomas of unknown primary origin (CUP) represent a diagnostic and therapeutic challenge. Squamous cell CUP located in the male pelvis are very rare. We describe a case of a locally advanced squamous cell CUP occurring in the male pelvis presenting as perineal abscess and urethral stenosis and diagnosed by means of transperineal needle biopsy.
\end{abstract}

KEY WORDS: Squamous cell carcinoma; Perineal abscess; Urethral stenosis.

Submitted 21 January 2017; Accepted 18 February 2017

\section{INTRODUCTION}

Carcinomas of unknown primary origin (CUP) represent a heterogeneous group of tumors for which a standardized diagnostic work-up fails to identify the site of origin and account for about 3-5\% of all malignancies (1). Squamous cell CUP account for 5-10\% of CUP and their occurrence in the male pelvic cavity is extremely rare (1). Initial presenting symptoms include buttock pain, rectal urgency, constipation, diarrhea, and urinary frequency (1). We describe a case of a locally advanced squamous cell CUP occurring in the male pelvic cavity and presenting as perineal abscess and urethral stenosis.

\section{Case report}

A 78-years old men presented to another hospital complaining of acute urinary retention, perineal pain and purulent perineal discharge. His past medical history was irrelevant. Transurethral catheterization of the bladder was impossible and cystoscopy revealed a severe urethral stenosis that made impossible the advancement of the instrument.

A perineal abscess secondary to urethral stenosis was suspected.

A suprapubic catheter was posi-

Figure 1. tioned, the perineal abscess was drained and a suspect urethrocutaneous fistula was excised. Histological examination showed a fibromuscular tissue with chronic inflammation and squamous epithelium. The patient was discharged home and a Magnetic Resonance Imaging (MRI) of the pelvis was prescribed. Six months later, the patient presented at our institution with intense perineal pain and persistent bleeding from the perineal surgical wound. Liver function tests were within the normal ranges. Serum creatinine level was $1.91 \mathrm{mg} / \mathrm{d}$. Physical examination revealed a bleeding perineal wound. Digital rectal examination was very painful and revealed a firm mass in the small pelvis and rectal bleeding. White blood cells count was within the normal range. Hemoglobin level was 9.0 g/dL. A contrast enhanced pelvic MRI showed a large $(11 \mathrm{~cm})$ inhomogeneous lesions with heterogeneous contrast enhancement infiltrating the prostate apex, the proximal corpora cavernosa, and the urethra (Figure 1).

Serum levels of CA 15-3, CA 19-9, CEA, CA-125, NSE tumor markers were within the normal range. Prostate Specific Antigen (PSA) level was $1.8 \mathrm{ng} / \mathrm{mL}$. A contrastenhanced total body computed tomography excluded other neoplasms.

Contrast-enhanced MRI of the pelvis demonstrating a $11 \mathrm{~cm}$ inhomogeneous pelvic lesion with heterogeneous contrast enhancement infiltrating the prostate apex, the proximal corpora cavernosa, and the urethra. A) transverse section, B) sagittal section.

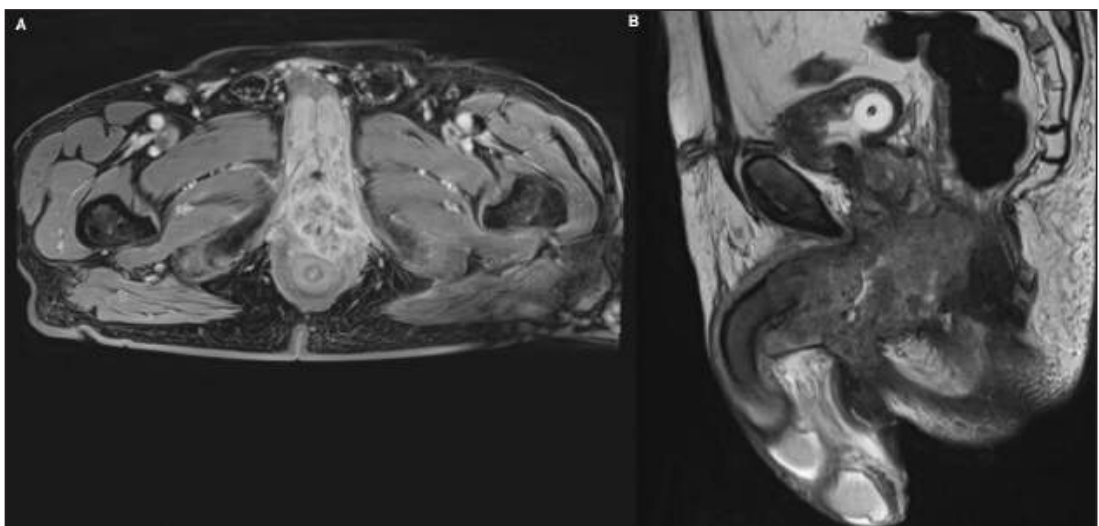

No conflict of interest declared. 
A colonscopy revealed an edematous rectal mucosa with signs of extrinsic compression.

Rectal biopsies were taken showing chronic inflammation and absence of malignancy. A transperineal needle biopsy of the pelvic mass was then performed showing fibro-muscular and adipose tissue with chronic inflammation and squamous cell carcinoma displaying solid tumor nests. Patients' general conditions rapidly worsened and he underwent colostomy for the urgent management of distal large bowel obstruction two months later. The patient was lost to follow-up after 3 months.

\section{Discussion}

CUP represent a diagnostic and therapeutic challenge. According to some authors, the criteria for CUP diagnosis include a biopsy-proven malignancy for which the anatomic origin is unknown after a medical history has been obtained, a detailed physical examination has been performed, and liver and kidney function tests, blood tests, chest radiography, abdomen and pelvis computed tomography, and a PSA test have been performed (2). Squamous cell CUP located in the male pelvis represent a rare entity, so the ideal diagnostic work-up, the optimal treatment strategies and prognosis are not well determined (1). In the present case, a primary anal neoplasm was unlikely as no anal lesions were noted on targeted biopsies. Moreover, other neoplasms were excluded on the basis of Computered Tomography and serum tumor markers results. Transperineal biopsy proved to be a valid option for histological diagnosis. In experienced hands, the transperineal route allows to access to the small pelvis in complex situations (3). However, we acknowledge that, due to the locally advanced disease, the diagnostic work-up was suboptimal. Indeed, urethral infiltration prevented to perform a detailed endoscopic study of the lower urinary tract. Moreover, due to the small amount of neoplastic tissue in the biopsy specimen, immunostaining was not performed. However, immunostaining is not generally helpful in differentiating metastatic squamous cell CUP (1). In general, CUP have a poor prognosis, with a median survival time of less than six months (1). Based on the limited literature data, it appears that squamous CUP outside of the head and neck region have a more favorable prognosis compared to other CUP and that these malignancies may respond well to a combination of surgical resection, when feasible, local radiotherapy, and platinum-based systemic chemotherapy (1). Due to the locally advanced disease and the rapidly worsened general conditions, the patient described in the present report could not undergo local surgical therapy or systemic chemotherapy.

\section{Conclusions}

Squamous cell CUP may arise in the male pelvic cavity and present, even in advanced stage, with non-specific signs and symptoms.

The present case underlines the misleading clinical course and the complex diagnostic work-up. Further studies are needed to determine ideal strategies for diagnosis, treatment, and prognostication of these neoplasms.

\section{References}

1. Chiec L, Verma S, Kendler A, Abdel Karim N. Male pelvic squamous cellcarcinoma of unknown primary origin. Case Rep Oncol Med. 2014; 2014:953698.

2. Varadhachary GR. Carcinoma of unknown primary origin. Gastrointest Cancer Res. 2007; 1:229-35.

3. Imperatore V, Creta M, Di Meo S, et al. Transperineal repair of a persistent rectourethral fistula using a porcine dermal graft. Int J Surg Case Rep. 2014; 5:800-2.

\section{Correspondence}

Massimiliano Creta, MD (Corresponding Author)

max.creta@gmail.com

Sergio Di Meo, MD

s.dimeo72@gmail.com

Roberto Buonopane, $\mathrm{MD}$

robertobuonopane@libero.it

Vittorio Imperatore, $\mathrm{MD}$

v.imperatore@alice.it

Unità Operativa di Urologia, Ospedale Buon Consiglio - Fatebenefratelli,

Napoli

Via A. Manzoni, 220 - 80123, Napoli, Italy

Vincenzo Mirone, MD, Professor of Urology

Nicola Longo, MD

Ferdinando Fusco, MD

Clinica Urologica, Università Federico II di Napoli

Via S.Pansini, 5, 80131 Napoli, Italy

Nicola Rosario Forte, MD

Unità Operativa di Anatomia Patologica, Ospedale Fatebenefratelli,

Benevento

Viale Principe di Napoli, 14, 82100, Benevento, Italy 